\title{
Betrachtung von neuartigen Antriebs- und Kraftstoffkonzepten mit dem Ziel der Konzeption einer Hybridtankstelle für den Flugplatz Schönhagen für die Versorgung von Luftfahrzeugen mit nachhaltigen Kraftstoffen
}

\author{
Andreas G. Hotes*, Klaus-Jürgen Schwahn und Henrike Fabienke
}

\section{Zusammenfassung}

Dieser wissenschaftliche Beitrag befasst sich zunächst mit der Betrachtung von neuartigen und nachhaltigen Antriebs- und Kraftstoffkonzepten für den Einsatz in der Luftfahrt, die zum Erreichen der hochgesteckten Klimaschutzziele auf nationaler, europäischer und internationaler Ebene beitragen sollen. Um die Energieversorgung dieser Luftfahrzeuge gewährleisten zu können, wurde ein Tankstellensystem beispielhaft für den Flugplatz Schönhagen konzipiert, welches Strom, Wasserstoff und Powerto-Liquid-Kraftstoff für Elektro-, Wasserstoff- und Luftfahrzeuge mit konventionellem Verbrennungsmotor sowie für Hybridflugzeuge zur Verfügung stellen soll. Ziel dieses Konzeptes ist die komplette Eigenproduktion der Kraftstoffe auf dem Flugplatz. Hierzu wurden rechtliche, wirtschaftliche und vor allem technische Voraussetzungen untersucht und ausgewertet sowie Berechnungen bezüglich der benötigten Kraftstoff- und Stromkapazitäten durchgeführt.

\section{Abstract}

This paper will initially focus on novel and sustainable propulsion and fuel concepts for use in aviation, which should contribute to the achievement of ambitious climate protection goals at national, European an international level. To ensure the energy supply of these aircrafts, a filling station system was designed as an example for the Schönhagen airfield, which provides electricity, hydrogen and power-to-liquid-fuel for electric and hydrogen driven aircrafts, aircrafts with conventional combustion engines as well as for hybrid aircrafts. The aim of this concept is the complete in-house production of fuels on the airfield. For this purpose, legal, economic, and above all technical requirements, were examined and evaluated and calculations were carried out regarding the required fuel and power capacities.

\section{Nachhaltigkeit in der zivilen Luftfahrt}

Die Nutzung eines Flugzeuges ist heutzutage nicht mehr aus unserem Alltag wegzudenken. Im Jahr 2017 wurden weltweit 4,1 Mrd. Passagiere (aero.de \& dpa-AFX 2018) innerhalb der kommerziellen Luftfahrt befördert. Doch Beachtung sollte man nicht nur dem rasanten Wachstum des Passagieraufkommens schenken, sondern auch dem Anteil der Luftfahrt am weltweiten $\mathrm{CO}_{2}$-Ausstoß. Dieser liegt bei $2 \%$ (International Air Transport Association IATA 2018: 2), wobei allerdings die negativen Auswirkungen auf die Umwelt durch die Höhenwirkung des Ausstoßes der Luftschadstoffe verstärkt werden (vgl. Pompl 2007: 57). Mit wachsendem Passagieraufkommen steigen darüber hinaus auch die luftverkehrsbezogenen Emissionen, sowohl was den Fluglärm als auch den Ausstoß von $\mathrm{CO}_{2}$ und Stickoxiden, etc. umfasst.

Um gerade diesem Wachstum der Schadstoffemissionen entgegenzuwirken und sie zu reduzieren, gibt es auf verschiedenen Ebenen unterschiedliche Ziele, Vorgaben und Projekte, die den Klimaschutz in der Luftfahrt vorantreiben sollen. Die International Civil Aviation Organization (ICAO) beschloss in einem Abkommen den sogenannten "Carbon neutral growth 2020“, das $\mathrm{CO}_{2}$-neutrale Wachstum der zivilen Luftfahrt ab dem Jahr 2020 (vgl. International Civil Aviation Organization IATA 2018). Um dieses Ziel umsetzen zu können, soll ab dem Jahr 2021 das Carbon Offsetting and Reduction Scheme for International Aviation, kurz CORSIA, eingeführt werden. In diesem Handelssystem erwerben u.a. die Fluggesellschaften, je nach Höhe ihres $\mathrm{CO}_{2}$-Austoßes, sogenannte 
Offsetting-Zertifikate, die wiederum Klimaschutzprojekte (vor allem in Entwicklungsländern) finanzieren, die in anderen Sektoren den $\mathrm{CO}_{2}$-Austoß verringern oder vermeiden und so einen Ausgleich schaffen (vgl. Bundesverband der Deutschen Luftverkehrswirtschaft BDL 2017: 11). Sowohl die Beteiligung in der Pilotphase als auch in Phase 1 des Offsetting-Handelssystems sind freiwillig.

Auch die Europäische Union (EU) hat sich hohe Klimaschutzziele gesteckt. Im „Flightpath 2050“ wird eine Reduktion von $\mathrm{CO}_{2}$ um $75 \%$, von Stickstoff und Stickoxiden um $90 \%$ und von Lärmemissionen um $65 \%$ angestrebt (vgl. Europäische Kommission 2011: 15). Innerhalb der EU ist bereits ein Handelssystem aktiv, das seit 2012 auch den Luftverkehr einschließt und bei dem Luftverkehrsunternehmen „European Union Allowances“ (EUA) kaufen müssen. Pro EUA wird es dem Unternehmen gestattet eine Tonne $\mathrm{CO}_{2}$ auszustoßen. Das europäische Emissionshandelssystem schließt allerdings Interkontinentalflüge aus (vgl. Fabienke 2018: 1), es wird nur für Flüge innerhalb der EU zur Anwendung gebracht.

Um die teils sehr ehrgeizigen Ziele auf internationaler und europäischer Ebene umsetzen zu können, reichen Emissions- und Offsetting-Handelssysteme allerdings nicht aus. Es muss mittelfristig auch eine Weiterentwicklung seitens der angewandten Technologien geben, die in der Luftfahrt eingesetzt werden. Hierzu gehören u.a. neuartige Antriebe für Luftfahrzeuge und moderne, weniger klimawirksame Kraftstoffe. Der Fokus der weiteren Betrachtung wird dabei auf die General Aviation (GA) gelegt, da der Einsatz von neuartiger Antriebstechnologie vor allem bei kleineren Luftfahrzeugen aus der GA zu finden ist und der Auf- und Ausbau der benötigten Infrastruktur für die Energieversorgung dieser Luftfahrzeuge dementsprechend auf kleineren Regionalflughäfen und Verkehrslandeplätzen schneller zu realisieren ist.

\section{Neuartige Antriebstechnologien}

Wie in Abb. 1 zu erkennen ist, liegt bei der Technologieentwicklung von Luftfahrzeugantrieben die Konzentration vor allem auf drei verschiedenen, neuartigen Antriebsmodellen. Eines ist der Antrieb durch einen Elektromotor mit Hilfe eines Akkusystems. Wird ein Luftfahrzeug mit ihm angetrieben, so wird die benötigte elektrische Energie in ein an Bord befindliches Akkusystem geleitet und in diesem gesammelt (vgl. Fabienke 2018: 7). Nachteilig ist hier aber vor allem die geringe Reichweite durch die geringe Energiedichte und das hohe Gewicht der Akkusysteme, die in der Luftfahrt vorrangig aus Lithium-Ionen- bzw. Lithium-PolymerAkkus bestehen. Zusätzlich existiert ein großer Nachteil in den verhältnismäßig langen Ladezeiten, die je nach Größe und Kapazität des Akkusystems variieren. Durch eine leicht mögliche operationelle Fehlbehandlung besteht eine relativ hohe Gefahr der Zerstörung des Akkusystems mit einer daraus resultierenden Brandgefahr. Wird als Energiequelle Strom aus erneuerbaren Energien wie Windkraft oder Solarenergie verwendet, ist es jedoch möglich, dass das Luftfahrzeug, lässt man die Emissionen während der Produktion z.B. der Solarzellen außen vor, emissionsfrei fliegen kann. Luftfahrzeuge mit Elektroantrieb sind bis jetzt nur vereinzelt in der General Aviation zu finden.
Wasserstoff entweder in gasförmiger oder flüssiger Form als Kraftstoff verwendet werden. Auch bei diesem neuartigen Antriebssystem besteht ein Gewichtsproblem. Obwohl Wasserstoff an sich leichter ist und eine höhere Energiedichte als z.B. Jet A1, umgangssprachlich Kerosin genannt, aufweist, benötigt er bis zu viermal mehr Raumvolumen. Durch zusätzlich benötigte Spezifikationen, wie ein spezielles Kühlsystem bei flüssigem und ein Drucktank bei gasförmigem Wasserstoff, tritt auch hier ein Gewichtsproblem auf, das allerdings durch die hohe Energiedichte und somit die große Reichweite größtenteils aufgehoben wird. Für das Modell eines Wasserstoffantriebes gibt es zwei mögliche Konfigurationen, die bis jetzt in der Luftfahrt betrachtet wurden bzw. aktuell betrachtet werden. In einem Mehrstofftriebwerk können entweder Jet A1 oder aber auch Wasserstoff konventionell verbrannt werden (vgl. KLS Publishing 2007: 8). Durch die hohe chemische Reinheit des neuartigen Kraftstoffs entstehen bei der Verbrennung keine Verbrennungsrückstände und nur geringe Mengen Stickoxide. Auch der Antrieb durch eine Brennstoffzelle ist möglich. Hierbei reagiert der Wasserstoff in der Zelle mit Sauerstoff zu Wasser. Dabei wird die chemische Reaktionsenergie in elektrische Energie umgewandelt, die anschließend einen Elektromotor antreibt und somit in mechanische Energie um-
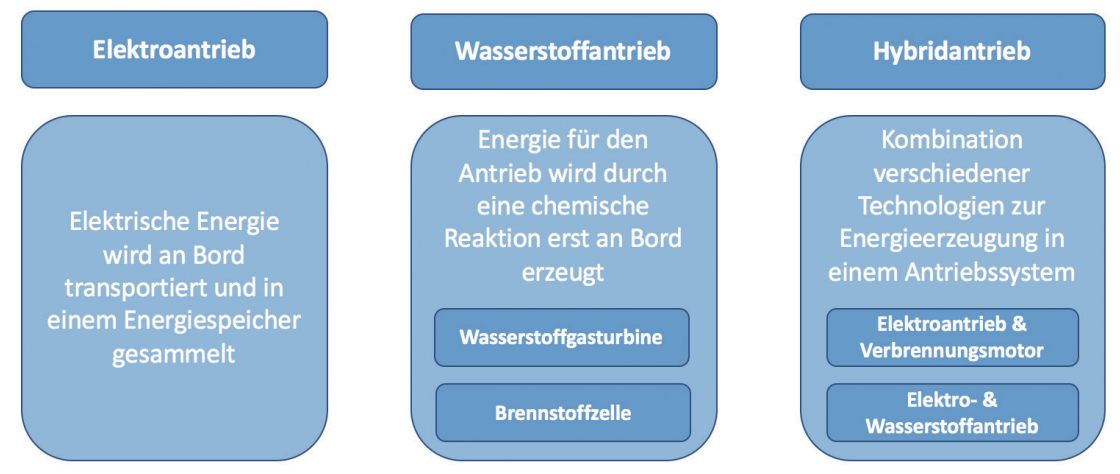

Abb. 1) Alternative Antriebsarten.

Ein weiteres Antriebsmodell, das zu einer Verbesserung der Nachhaltigkeit in der Luftfahrt führen soll, ist der Wasserstoffantrieb. Hier wird die Energie, die für den Antrieb des Luftfahrzeugs benötigt wird, erst an Bord des Luftfahrzeuges durch eine chemische Reaktion produziert. Hierbei kann der gewandelt wird. Die Reaktion erfolgt effizient und ohne die Entstehung schädlicher Abgase. Luftfahrzeuge mit reinem Wasserstoffantrieb fliegen zurzeit nur mit Experimentalzulassung und werden für Forschungszwecke eingesetzt. Als drittes Antriebsmodell besteht die Möglichkeit einer Hybridisierung von 
zwei einzelnen Antrieben. So werden verschiedene Technologien zur Energieerzeugung in einem Antriebssystem kombiniert. Gängige Kombinationen sind zum einen die Paarung aus Elektro- und Verbrennungsmotor sowie die Paarung aus Elektro- und Wasserstoffantrieb, wobei für Letztere zumeist ein Brennstoffzellensystem eingesetzt wird. Bei der Hybridisierung werden vor allem die Vorteile der zwei unterschiedlichen Möglichkeiten zusammengeführt. Meist dient einer der Antriebe als Reichweitenverlängerung (Range Extender) und/oder als Unterstützung für den Start und den Steigflug.

Die vorgestellten neuartigen Antriebstechnologien werden in Zukunft eher bei kleineren Flugzeugen in der General Aviation oder bei den kleineren Luftfahrzeugen in der kommerziellen Regionalluftfahrt zu finden sein. Denn große Langstreckenluftfahrzeuge haben einen höheren Energiebedarf, der nach dem aktuellen Stand der Technik weiterhin nur durch flüssige Kraftstoffe abgedeckt werden kann. Auch haben Flugzeuge sehr lange Nutzungszyklen von 30 Jahren und mehr. Aufgrund dieser langfristigen Nutzungsdauer ist eine Übergangsphase von 30-50 Jahren vorzusehen, in der das herkömmliche Kerosin trotz neuer Energieträger weiterhin parallel Verwendung finden wird.

\section{Alternative Kraftstoffarten}

Um auch mit großen Luftfahrzeugen emissionsarm oder zukünftig emissionsfrei fliegen zu können, ist in diesem Bereich der Einsatz von alternativen Kraftstoffen sinnvoll. Unter alternativen Kraftstoffen werden diejenigen Kraftstoffe aufgeführt, die in der Rohstoffbasis eine Alternative zum konventionellen Rohöl darstellen. Alternative Kraftstoffe aus fossilen Energieträgern, wie Erdgas und Kohle, sollen hier nur am Rande erwähnt werden, da aus ihnen zwar ebenfalls Kraftstoff hergestellt werden kann, doch damit keine relevante Emissionsminderung einhergeht.

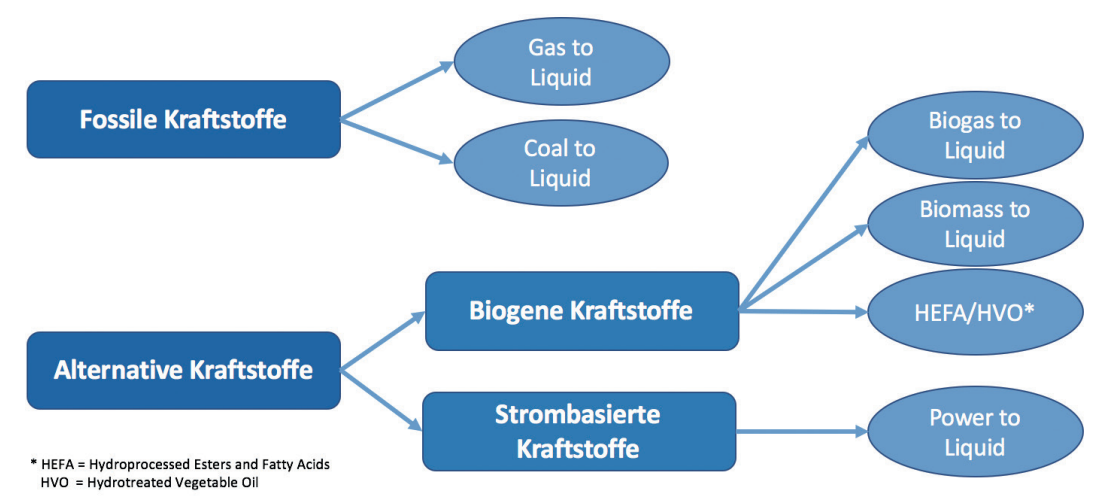

Abb. 2) Alternative Kraftstoffarten.

Besonders zukunftstragend sind synthetische Kraftstoffe, die in Abb. 2 dargestellt sind. Biogas-to-Liquid-, Biomass-to-Liquid- und HEFA/HVOKraftstoffe werden aus unterschiedlichen biogenen Rohstoffen (z.B. Biogas, Energiepflanzen, Rest- und Abfallstoffen aus Forst- und Landwirtschaft) hergestellt. Die Kraftstoffe werden bei der Verbrennung als $\mathrm{CO}_{2}$-neutral angesehen, da die verwendeten Rohstoffe beim Anbau so viel $\mathrm{CO}_{2}$ binden, wie bei der Verbrennung entsteht. Während des Herstellungsprozesses (Verarbeitung, Transport, Vertrieb) werden allerdings Treibhausgasemissionen erzeugt. Diese liegen jedoch deutlich unter den Werten der fossilen Kraftstoffe und stellen so eine eindeutige Verbesserung bezüglich ihrer klimaschonenden Wirkung gegenüber diesen dar. Doch bei der Rohstoffgewinnung kann es bei biogenen Kraftstoffen zur Konkurrenz mit dem Nahrungsmittelanbau oder anderen Industriezweigen (z.B. Papierindustrie) kommen. Werden statt Abfall- und Reststoffen Energiepflanzen als Rohstoffbasis verwendet, besteht die Gefahr einer negativen Landnutzungsänderung, d.h. z.B. der Umwandlung einer vorhandenen Agrarfläche für den Anbau von Nahrungsmitteln in eine Agrarfläche für den Anbau von Energiepflanzen.

Doch vor allem dem zweiten Pfad der alternativen Kraftstoffe in Abb. 2 ist Aufmerksamkeit zu schenken. Die strombasierten Kraftstoffe, Power-to-Liquids (PtL) genannt, stechen vor allem durch ihre minimalen Emissionswerte sowohl bei der Verbrennung als auch bei der Herstellung hervor. Für die Herstellung gilt das allerdings nur, wenn der Strom aus erneuerbaren Energien (Windkraft,
Wasserkraft, Solarenergie) erzeugt wurde. Bei der Verbrennung wird PtL, genauso wie sein biogenes Pendant, als $\mathrm{CO}_{2}$-neutral angesehen, da während der Kraftstoffsynthese so viel $\mathrm{CO}_{2}$ aus der Umgebungsluft oder anderen $\mathrm{CO}_{2}$-Quellen entnommen wird, wie bei der Verbrennung entsteht. Weitere Vorteile von PtL bestehen darin, dass die Konkurrenz zum Nahrungsmittelanbau nicht existiert, eine starke politische Unterstützung der Bundesregierung durch den Beschluss der Förderung von Forschung und Entwicklung von strombasierten Kraftstoffen im Koalitionsvertrag festgelegt wurde und zum jetzigen Zeitpunkt die Beimischung von PtL-JetA1-Flugkraftstoff schon zu 50 \% möglich ist (vgl. Fabienke 2018: 57). Auch die Technologie für die Herstellung von PtL ist bereits vorhanden. Die Power-to-Liquid-Produktion kann in drei Hauptschritte unterteilt werden: Zunächst erfolgt die Stromgewinnung aus erneuerbaren Energien. Anschließend wird dieser gewonnene Strom mit Hilfe einer Elektrolyse-Einheit in Wasserstoff umgewandelt, der wiederum im nachfolgenden Schritt mit Kohlenstoffmonoxid (gewonnen aus $\mathrm{CO}_{2}$ z.B. aus der Luft) in einem chemischen Reaktor zu dem gewünschten Endprodukt synthetisiert wird.

\section{Tankstellensystem}

\subsection{Anforderung}

Um die vorgestellten neuartigen Antriebstechnologien und Kraftstoffe etablieren zu können, muss vor allem darauf geachtet werden, die passende und ebenfalls neuartige Infrastruktur bereitstellen zu können. Daher wurde beispielhaft ein Tankstellensystem 
für den Flugplatz Schönhagen konzipiert, welches genau diese benötigte Infrastruktur abbilden kann und nachfolgend vorgestellt werden soll. Der Flugplatz Schönhagen ist, bezogen auf die Infrastruktur und die Anzahl der Flugbewegungen, einer der größten Verkehrslandeplätze in Deutschland und für die Flughäfen im Berliner Raum ein wichtiger Entlastungsstandort (vgl. Flugplatz Schönhagen 2018a) für die Luftfahrzeugkategorie bis $14 \mathrm{t}$ Maximum Take Off Weight (MTOW). Der Flugplatz beteiligt sich u.a. am „Innovationsbündnis für die Entwicklung emissionsarmer Flugantriebe in der südöstlichen Metropolregion BerlinBrandenburg" (IBEFA) für einen zukunftsorientierten, nachhaltigen Flugplatzbetrieb im Rahmen des Projektes "Airport of the Future“.

Das Tankstellensystem sollte folgenden Herstellungsumfang abbilden können: Zum einen wird die Gewinnung von Strom aus erneuerbaren Energien für die Versorgung von Luftfahrzeugen mit Elektroantrieb genutzt. Aus überschüssigem Strom soll weiterführend Wasserstoff in einer Elektrolyse-Einheit für die Versorgung von Wasserstoffflugzeugen produziert werden. Überschüssiger Wasserstoff wird dann abschließend für die Power-to-Liquid-Produktion genutzt, um auch Luftfahrzeuge mit konventionellen Verbrennungsmotor das emissionsarme Fliegen ermöglichen zu können. Ziel dieses Vorhabens ist die komplette flugplatzeigene Produktion des Kraftstoffes.

\subsection{Konzept}

Die Aufteilung des Tankstellensystems orientiert sich z.T. an den Teilschritten der Power-to-Liquid-Produktion. So entstehen drei einzelne Systembausteine, die in Abbildung 3 dargestellt sind: die Energiegewinnungsanlage, die Herstellungsanlage und die Tankstelle, die alle miteinander verbunden sind. In der Hauptarbeit erfolgte keine umfassende Auslegung des Tankstellensystems an sich. Es wurde stattdessen ein Überblick über aktuelle und grundlegende Möglichkeiten gegeben, die bei einem Tankstellensystem dieser Art eingesetzt werden könnten. Dementsprechend findet auch in diesem Beitrag nur eine überschlägige Betrachtung der

Auslegung statt. In den Vordergrund gestellt wird hier die Kapazitätsberechnung und die Betrachtung der grundsätzlichen Machbarkeit.

von $10.000 \mathrm{~m}^{2}$ Photovoltaikanlagen abgedeckt werden. Beim Einsatz dieser ist aber vor allem darauf zu achten, dass die Gefährdung des Flugbetriebs durch

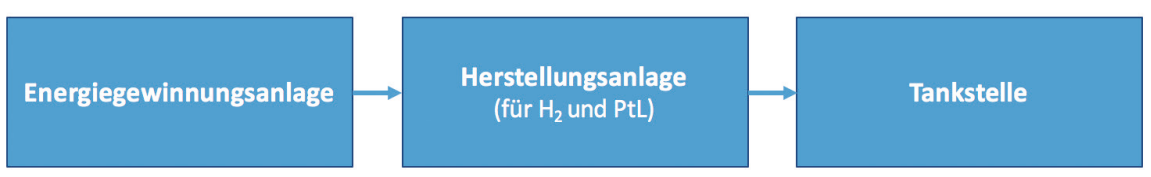

Abb. 3) Bausteine des Tankstellenkonzeptes.

Auf dem Flugplatz Schönhagen werden jährlich ca. 152.000 kWh Strom für die Flugplatzinfrastruktur (Gebäude, Straßen- und Parkplatzbeleuchtung, Tankstelle, Befeuerung Start- und Landebahn) verbraucht. Geht man nun davon aus, dass bei einer Gesamtzahl von 60.000 prognostizierten Flugbewegung für das Jahr 2028 etwa $15 \%$ von Luftfahrzeugen mit Elektroantrieb (Hybridflugzeuge einbezogen) absolviert werden, ergibt sich bei verschiedenen Flugzeugtypen mit unterschiedlichen Akkukapazitäten (21-900 kWh) ein Jahresbedarf von ca. 415.000 kWh Strom. Hinzu kommt zusätzlich der Strombedarf für die PtL-Produktion. Um ein 10\% PtL-Beimischungsverhältnis realisieren zu können, werden bei einem angenommenen Kraftstoffbedarf für das Jahr 2028 von 300.000 I JetA1 30.000 I PtL-JetA1 benötigt. Für dieses Produktionsvolumen ergibt sich ein Strombedarf von ca. 430.000 kWh (bei einem Wirkungsgrad der Produktionsanlage von 50\%). Daraus folgt, dass pro Jahr etwa 1 Mio. kWh Strom auf dem Flugplatz zur Verfügung gestellt werden müssten. Hieraus ergibt sich die erste Problemstellung: Welche Energiegewinnungsanlage kann diesen hohen Strombedarf mit der Eigenproduktion decken?

Auf dem Flugplatz Schönhagen stehen mehr als $10.000 \mathrm{~m}^{2}$ freie Dachfläche zur Verfügung. Zusätzlich könnten große Freiflächen z.B. zwischen den zwei Start- und Landebahnen als Standort für die Energiegewinnungsanlage genutzt werden. Aufgrund dessen ist der Einsatz von Photovoltaikanlagen (PV-Anlagen) eine gute Möglichkeit. Geht man davon aus, dass im Idealfall pro $\mathrm{m}^{2}$ Solarmodul $100 \mathrm{kWh}$ Strom pro Jahr erzeugt werden könnten, könnte der errechnete Gesamtstrombedarf
Blendung der Piloten, Funkstörung, etc. ausgeschlossen wird.

Dem Ziel der kompletten Eigenproduktion zuwider stellt sich trotzdem die Frage, ob der Zukauf von Strom nicht doch eine mögliche Ergänzung darstellt, gerade da die Stromproduktion durch erneuerbare Energien mit starken, umwelt- bzw. wetterbedingten Schwankungen verbunden ist. So sinken die Investitionskosten der PVAnlage, gleichzeitig entstehen allerdings neue Kosten für den zugekauften Strom. Im letzten Jahr betrug der durchschnittliche Strompreis 0,29 €/ kWh. Geht man davon aus, dass die Hälfte des für die $10 \%$ PtL-Produktion benötigten Stroms durch den Zukauf abgedeckt wird, wirken sich diese Stromkosten allerdings mit 2,10 € auf den späteren Literpreis des Kraftstoffes aus, alleine für den zugekauften Teil. Der aktuelle Preis von fossilem JetA1 (steuerbefreit und ohne 19\% Mehrwertsteuer) liegt vergleichsweise bei $1,08 €$. Möchte man diesen aktuellen Wert beispielhaft als Kostenanteilszielwert des zugekauften Stroms für die Produktion von synthetischem JetA1 realisieren, müsste der Strompreis bei ca. $0,15 € / k W h$ liegen, also deutlich unter dem Durchschnittsstrompreis aus 2017. Hinzu kommt hier allerdings noch der Kostenanteil für die flugplatzeigene Stromproduktion. Würde dieser ebenfalls bei $0,15 €$ liegen, ergibt sich eine Gesamtauswirkung von ungefähr $2,20 €$ auf den späteren Kraftstoffpreis des geblendeten JetA1, alleine durch die anfallenden Stromkosten. Je kleiner die Stromkosten, desto kleiner natürlich auch die Auswirkungen auf den späteren Kraftstoffpreis. Aufgrund dessen muss der Strompreis deutlich unter den $0,15 €$ liegen, um die Konkurrenzfähigkeit des nachhaltigen Kraftstoffes 
zu gewährleisten. Allerdings muss man bei diesen Überlegungen berücksichtigen, dass hier an einer Technologie für die Zukunft gearbeitet wird. Die Wirtschaftlichkeit wird erst mittelfristig in einer Serienproduktion eine Rolle spielen. Bis dahin werden die Kosten für fossile Energieträger deutlich gestiegen sein. Bedenkt man, dass ein Liter Jet $A 1$ versteuert heute am Flugplatz Schönhagen 2,06€ kostet und ein Liter AVGAS 2,41€ (Flugplatz Schönhagen 2018b) würden in der Forschungs- und Erprobungsphase bereits Steuererleichterungen helfen, um den PtL-Kraftstoff auch tatsächlich in der Praxis einsetzen zu können. Bei dieser Preisbetrachtung ist allerdings noch anzumerken, dass hier weitere anfallende Kosten wie z.B. Kosten für die Beschaffung, den Betrieb und die Instandhaltung der benötigten Infrastruktur (u.a. Produktionsanlagen, Photovoltaikanlagen, Personalkosten) vereinfachend nicht berücksichtigt wurden.

Bei der Herstellungsanlage besteht die wichtigste Voraussetzung darin, dass die Anlage erweitert werden kann. Je nach steigendem Beimischungsverhältnis, das in Tab. 1 abgebildet ist, steigt der Bedarf an PtL-JetA1. Deshalb muss das Anlagensystem vorzugsweise bis zur jetzigen Zertifizierungsgrenze von 50 \% Beimischung (in Tab. 1 gelb hinterlegt) „mitwachsen“ können. Anlagen, die die PtL Herstellung komplett durchgängig durchführen können sind noch nicht kommerziell verfügbar. Demonstrationsanlagen hingegen schon, die je nach Art und Bauweise 80-160 Liter pro Tag des gewünschten Endproduktes herstellen können. Mit diesen Anlagen könnte man bereits eine Beimischung von 10-20 \% realisieren.

Interessant wäre in diesem Zusammenhang der Einsatz von PtL-Kraftstoffen in Flugzeugen mit Hybridantrieb. Wenn z.B. eine kleine Turbine oder ein Verbrennungsmotor nur der Stromerzeugung im Flugzeug dienen und der eigentliche Antrieb über einen Elektromotor erfolgt, könnte u.U. die $50 \%$ Blendinggrenze entfallen, d.h. ein PtLKraftstoff zu $100 \%$ eingesetzt werden. Auf diese Weise hätte man schon heute einen quasi $\mathrm{CO}_{2}$ neutralen Antrieb.

Die Herstellungsanlage besteht ebenfalls aus mehreren Komponenten. Neben der Elektrolyse-Einheit für die Wasserstoffproduktion und der Synthese-Einheit für die finale Kraftstoffherstellung gehören auch eine Blendinganlage für das Mischen des fossilen mit dem synthetischen JetA1 und das Distributionssystem für den Transport des geblendeten Kraftstoffs zur Tankstelle zum Herstellungssystem. Für die Realisierung des Letzteren empfiehlt sich ein unterirdisches Leitungssystem, das die Herstellungsanlage direkt mit der Tankstelle verbindet.
Die derzeitige Tankstelleninfrastruktur (Lagerungssystem und Zapfsäule) für fossiles JetA1 auf dem Flugplatz selbst kann für die Betankung mit PtL-JetA1 beibehalten werden. Dies ist ein weiterer Vorteil des strombasierten Kraftstoffs. Um zusätzlich Elektroflugzeuge mit Strom versorgen zu können, empfiehlt sich eine Auslagerung der benötigten Ladestationen, um die Kapazitäten der eigentlichen Tankstelle aufgrund der langen Ladezeiten der Akkusysteme nicht zu überlasten. Zum einen sollten mehrere Ladestationen in den Hangars verteilt werden, um für Langzeitmieter eines Stellplatzes einen einfachen Zugang zum Strom zu schaffen. Für Tages- oder Kurzzeitgäste wäre es sinnvoll, einige Ladestationen an ausgewählten Abstellplätzen auf dem Vorfeld zu platzieren. Bei den Ladestationen wird es allerdings zusätzlich nötig sein, sowohl den Ladeprozess an sich als auch die Vorrichtungen mit den zugehörigen Steckern und den unterschiedlichen Stromstärken zu standardisieren. Die Tankinfrastruktur für die Wasserstoffversorgung von Luftfahrzeugen (Zapfsäulen- und Speicherungssystem) muss ebenfalls geschaffen werden. Eventuell einzuhaltende Sicherheitsmaßnahmen wie z.B. Sicherheitsabstände zu Zündquellen wie den anderen Kraftstoffen müssen vor und vor allem bei der Implementierung in das bestehende Tanksystem beachtet werden. Auch hier empfiehlt sich eine Standardisierung des Tankprozesses

Tab. 1) Benötigte Mengen von synthetischem PtL- und fossilem JetA1 für verschiedene Beimischungsverhältnisse (Grenze des aktuell zertifizierten Beimischungsverhältnisses orange hinterlegt).

\begin{tabular}{|c|c|c|c|c|c|c|}
\hline $\begin{array}{l}\text { Beimischungs- } \\
\text { verhältnis von } \\
\text { synthetischem und } \\
\text { fossilem JetA1 [\%] }\end{array}$ & $\begin{array}{c}\text { Bedarf an } \\
\text { synthetischem } \\
\text { PtL-JetA1 pro } \\
\text { Jahr [l] }\end{array}$ & $\begin{array}{c}\text { Bedarf an } \\
\text { synthetischem } \\
\text { PtL-JetA1 pro } \\
\text { Monat [I] }\end{array}$ & $\begin{array}{l}\text { Bedarf an syn- } \\
\text { thetischem PtL- } \\
\text { JetA1 pro Tag } \\
\text { [l] (gerundet) }\end{array}$ & $\begin{array}{c}\text { Bedarf an fos- } \\
\text { silem JetA1 pro } \\
\text { Jahr [I] }\end{array}$ & $\begin{array}{l}\text { Bedarf an fos- } \\
\text { silem JetA1 pro } \\
\text { Monat [I] }\end{array}$ & $\begin{array}{c}\text { Bedarf an } \\
\text { fossilem JetA1 } \\
\text { pro Tag [l] } \\
\text { (gerundet) }\end{array}$ \\
\hline $10: 90$ & 30.000 & 2.500 & 82 & 270.000 & 22.500 & 740 \\
\hline $20: 80$ & 60.000 & 5.000 & 164 & 240.000 & 20.000 & 657 \\
\hline $30: 70$ & 90.000 & 7.500 & 247 & 210.000 & 17.500 & 575 \\
\hline $50: 50$ & 150.000 & 12.500 & 411 & 150.000 & 12.500 & 411 \\
\hline $80: 20$ & 240.000 & 20.000 & 657 & 60.000 & 5.000 & 14 \\
\hline 100:0 & 300.000 & 25.000 & 822 & 0 & 0 & 0 \\
\hline
\end{tabular}


und des Tanksystems. Vor allem bei Wasserstoff sollte ein besonderes Augenmerk auf die Sicherheitsvorkehrungen und Notfallpläne gelegt werden.

\subsection{Diskussion}

Aus dem vorgestellten Tankstellensystem ergeben sich zwei große Defizite. Zum einen existieren noch keine Gesetze, Vorschriften und/oder Normen, die sich explizit mit der Verwendung von Wasserstoff in Verbindung mit Luftfahrzeugen und mit dem Handling des Wasserstoffes auf Flugplätzen befassen (vgl. Fabienke 2018: 91). Unter den letzten Punkt fallen auch die Wasserstoffproduktion und der Wasserstofftankprozess auf dem Flugplatz. Aus diesen Defiziten leitet sich der Bedarf nach rechtlichen Vorgaben ab, die u.a. Sicherheitszonen und -abstände, Anforderungen an die benötigte Infrastruktur, den Ablauf des Tankprozesses etc. gesetzlich regeln.

Zum anderen besteht ein weiteres Problem darin, dass das Blenden von Kraftstoffen direkt auf dem Flugplatz innerhalb der Europäischen Union nicht gestattet ist (vgl. Fabienke 2018: 98). Dieser Prozess ist allerdings essentiell für den Erfolg und die Erhaltung der Wirtschaftlichkeit dieses Projektes, denn die Distributionskosten des geblendeten Kraftstoffes müssen so gering wie möglich gehalten werden. Muss der produzierte PtL-Kraftstoff erst zu einer externen Blendinganlage gebracht werden, um danach wieder zurücktransportiert zu werden, wären erhebliche Kosten und Risiken die Folge. Erfolgt weiterhin keine Erlaubnis des Blendens auf dem Flugplatz, dann wäre einzig und allein mit einem Standort der Blendinganlage außerhalb des Flugplatzgeländes allerdings in unmittelbarer Nähe die einzige Möglichkeit, um den Distributionsweg so kurz wie möglich zu halten und somit das Projekt erfolgreich und wirtschaftlich realisieren zu können.

\subsection{Bewertung der Machbarkeit}

Die Machbarkeit des Tankstellensystems ist im Wesentlichen von drei Punkten abhängig:

1. Das Finden von Projektpartnern und Investoren: Diesem Punkt kommt eine besondere Bedeutung zu, denn die Investoren müssen den noch unbekannten Kostenrahmen tragen können und wollen.

2. Staatliche Förderung der Forschung und Entwicklung von strombasierten Kraftstoffen in Form von Projektgeldern und später durch Subventionen: Beispielhaft könnte man hier die Energiesteuer je nach prozentualem Beimischungsverhältnis für PtL-JetA1 um denselben Prozentsatz senken.

3. Behebung der juristischen Genehmigungs-Defizite: Hierzu gehört, zum einen die Nichtexistenz von Vorgaben für den Umgang mit Wasserstoff auf Flugplätzen und zum anderen der fehlenden Erlaubnis des Blendens von Kraftstoffen auf dem Flugplatz.

Viele Faktoren aus technischer und sicherheitstechnischer Sicht, wie z. B. das Vorhandensein der benötigten Technologie oder die Erweiterung von bereits existierenden Sicherheitsprogrammen und -abläufen könnten hingegen heute schon problemlos erfüllt werden.

\section{Zusammenfassung}

Für die Umsetzung der ehrgeizigen Ziele der Luftfahrtbranche und der Politik auf nationaler, europäischer und internationaler Ebene ist es notwendig, dass neuartige Antriebstechnologien wie Elektro-, Wasserstoff- oder Hybridantriebsmodelle entwickelt werden. Auch für Luftfahrzeuge mit konventionellem Verbrennungsmotor ist eine nachhaltige alternative Antriebsversorgung durch biogene oder strombasierte Kraftstoffe gegeben. Letztere stechen durch ihre vielen positiven Eigenschaften wie die $\mathrm{CO}_{2}$-Neutralität sowohl bei der Produktion als auch bei der Verbrennung und der bereits vorhandenen $50 \%$ Beimischungszertifi- zierung für JetA1 hervor. Doch für die Kraftstoffversorgung mit diesen neuen Technologien muss die passende Infrastruktur bereitgestellt werden können. Überwindet man die vor allem rechtlichen und finanziellen Hürden des Projektes, ist es möglich, auf dem Flugplatz Schönhagen eine Hybrid-Tankstelle für die Versorgung von Elektroflugzeugen mit Strom, von Wasserstoffflugzeugen mit Wasserstoff und von Luftfahrzeugen mit Verbrennungsmotor mit PtLKraftstoff zu realisieren.

Es existiert eine Vielzahl an Projekten für alternative Antriebs-, Luftfahrzeugund Kraftstoffkonzepte. Diese spiegeln u.a. auch den Trend und das Streben nach Nachhaltigkeit in der Luftfahrtbranche wieder. Deshalb ist es vor allem wichtig, Projekte wie das hier vorgestellte Tankstellenkonzept voranzutreiben, um die benötigte neuartige Infrastruktur zur Verfügung zu stellen und so ein Bewusstsein für neue und nachhaltige Konzepte zu schaffen und diese in der Luftfahrt zu etablieren. 


\section{LITERATUR}

aero.de, dpa-AFX (2018) Zahl der Flugpassagiere 2017 au Rekordniveau. https://www.aero.de/news-28401/Zahlder-Flugpassagiere-2017-auf-Rekordniveau.html. Accessed 13 Dec 2018

Bundesverband der Deutschen Luftverkehrswirtschaft BDL. Keine Zukunftsmusik: Kerosin aus Wasser und $\mathrm{CO}_{2}$. https://www.klimaschutz-portal.aero/klimaneutral-fliegen/alternative-kraftstoffe/kerosin-aus-wasser-und-co2/. Accessed 14 Dec 2018

Bundesverband der Deutschen Luftverkehrswirtschaft BDL (2017) CORSIA: Globales marktbasiertes Klimaschutzinstrument für den internationalen Luftverkehr. Positionspapier

Europäische Kommission (2011) Flightpath 2050. Europe‘s vision for aviation ; maintaining global leadership and serving society's needs; report of the High-Level Group on Aviation Research. Policy / European Commission. Publ. Off. of the Europ. Union, Luxembourg.

ISBN: 978-92-79-19724-6

Fabienke H. (2018) Nachhaltigkeit in der zivilen Luftfahrt - Betrachtung von alternativen Antriebs-, Kraftstoff- und Luftfahrzeugkonzepten mit dem Ziel der Konzeption eine Hybrid-Tankstelle auf dem Flugplatz Schönhagen für die Energieversorgung mehrerer Antriebsarten. Bachelorarbeit, Technische Hochschule Wildau

Flugplatz Schönhagen (2018a) Herzlich willkommen auf dem Flugplatz Schönhagen. http://www.flugplatz-schoenhagen.aero/der-flugplatz.html. Accessed 13 Dec 2018

Flugplatz Schönhagen (2018b) Treibstoffpreise. http:// www.flugplatz-schoenhagen.aero/piloten/verkehrslandeplatz/treibstoffpreise.html. Accessed 14 Dec 2018

International Air Transport Association IATA (2018) Fact Sheet: Climate Change \& CORSIA.

International Civil Aviation Organization ICAO. Why ICAO decided to develop a global MBM scheme for international aviation? https://www.icao.int/environmental-protection/pages/a39_corsia_faq1.aspx. Accessed 14 Dec 2018

KLS Publishing (2007) Fliegen mit Wasserstoff. Eine Bestandsaufnahme. http://klspublishing.de/ejourns/eJourn\%20Al-04\%20Fliegen\%20mit\%20Wasserstoff.pdf. Accessed 13 Dec 2018

Pompl W (2007) Luftverkehr. Eine ökonomische und politische Einführung, 5., überarb. Aufl. Springer-Lehrbuch. Springer-Verlag Berlin Heidelberg, Berlin, Heidelberg. ISBN: 9783540327523 . doi: 10.1007/978-3-540-32753-0

\section{AUTOREN}

Andreas G. Hotes, Dipl.-Ing Henrike Fabienke, B.Eng.

Fachgebiet Airlinemanagement \& Aviation Security

Studiengang Luftfahrttechnik / Luftfahrtlogistik

Fachbereich INW

Technische Hochschule Wildau

Dr.-Ing. Klaus-Jürgen Schwahn

Flugplatzgesellschaft Schönhagen mbH

E-Mail für Korrespondenz:

andreas.hotes@th-wildau.de 